\title{
Project Clustering and Risk Monitoring based on QFD and MDM
}

\author{
Qinru Wang ${ }^{1}$, Qing Yang*1, Mingxing Chang ${ }^{1}$, Tao Yao $^{2}$ \\ ${ }^{1}$ School of Economics and Management, University of Science and Technology Beijing, \\ China \\ ${ }^{2}$ Alibaba US, 500 108th Ave NE \#800, Bellevue, WA 98004, USA
}

\begin{abstract}
Projects are faced with various complex risks, and how to cluster management of projects with high risk management has become very important. Therefore, this article first constructs the dependency structure matrix (DSM) between risk events and risk factors, and uses the Quality Function Development (QFD) method to derive the risk event DSM matrix, and obtains the dependency relationship model between risk events; then, builds the risk dependency relationship between the projects, through risk the event-project MDM derives the project DSM; finally, the project DSM is optimized and analyzed by the clustering method, and the dependence intensity between the projects is calculated, and the project set with high risk correlation is obtained. This paper calculates the dependencies between projects from the perspective of risk, and clusters them according to the dependencies between the projects, and monitors the risks of the projects by gathering the projects with high risk dependence.
\end{abstract}

Keywords: Risk management, Project management, DSM (Dependency Structure Matrix), MDM (multi-domain Matrix), QFD (Quality Function Development).

\section{Introduction}

From the perspective of project management, risk can be understood as a potential threat to the project once it occurs, and it can also be understood as an uncertain factor that may have an impact on the entire process of the project. Large and medium-sized engineering projects generally have large investments and long periods. In the process of construction and production, they are often affected and interfered by many factors, and most of these factors have considerable uncertainty, and the impact time span often runs through the entire life of the project. The cycle, that is, from the pre-feasibility study stage to the end of production after the project is put into production, often results in investment decisionmaking errors, poor construction plan planning, construction period delays, personal injury, property damage, abnormal production and operation, and low investment returns or even losses and other serious consequences.

There are two main reasons for the complexity of the project. First, the dynamic nature of the project that is, due to differences in the project cycle and project status, the dynamic change of the project in different cycles is triggered. The status of the project mainly includes four statuses: up, running, completed, and failed. Second, the diversity of the project relationship, that is, the various interactive relationships between projects, which 
mainly include interactions in terms of income, resources, and technology. Under the effect of this structural complexity, the project risks in the project are related to each other. Once one or more projects fail, other related projects will be affected through this correlation, and the risks of these projects will be changed, thereby triggering more the failure of the project may not only lead to failure in the implementation of the corporate strategy, but even more serious, may cause the enterprise to go bankrupt. In view of this, it is particularly important for business managers to accurately analyze the risk-related issues of project caused by this structural complexity.

\section{Literature Review}

The basis and premise of engineering project risk management is risk identification. Risk identification is to define and identify various risk sources or uncertain factors in the project according to their background reasons, performance characteristics and expected consequences, and to scientifically classify all risk factors in order to adopt different analyses (Kim et al, 2018). Methods are evaluated, and corresponding risk management plans and measures are formulated based on this, and put into practice.

There are several commonly used methods (Neumeier et al., 2011).

1. Expert survey method

The expert investigation method includes the expert personal judgment method, the outrageous method and the Delphi method. This type of method mainly uses the professional theories and rich practical experience of experts in various fields to find out various potential risks and analyze and estimate the consequences.

2. Fault tree analysis method

The fault tree analysis method is also called the causal analysis graph method. This method uses a graphical form to decompose large faults into various small faults, or analyze various causes of the faults.

3. Checklist method

The risk checklist is compiled by the relevant personnel based on their knowledge. The advantage of the checklist method is that it can make risk identification more systematic, standardized, simple and easy to implement; the disadvantage is that the checklist cannot be all-encompassing and has certain limitations.

According to the difference in the structure of the project and its external environment, project dependencies are mainly divided into internal dependencies and external dependencies (Aerts et al., 2016). The internal dependency mainly refers to the change in the income, resource requirements, or success probability of a project during the formation of the project, which depends on the selection or exclusion of one or more projects; the external dependency refers to the transition in time. In the process of progress, the extent to which the project changes with changes in the external social and economic environment. The focus of this paper is the internal dependencies between projects. The impact of project dependencies on risk is divided into two aspects. On the one hand, due to the existence of inter-project dependencies, the cost, benefit, and success probability of 
a project depend to a large extent on whether other projects are selected. This dependency has an impact on each item in the project (Ghapanchi et al, 2012). Risks have an important impact, leading to differences in the sum of project risks, which in turn changes the risks assumed by the project (Aerts et al., 2016). Therefore, project dependencies affect the risk of the project. On the other hand, the dependencies of projects originate from the overlapping requirements of resources, benefits, and technologies between projects. The size of the set of neighbouring project dependencies of a project in a project will also affect the project dependencies, which in turn has an impact on project risks.

The design structure matrix (DSM) was proposed and researched by the famous scholar Steward in the 1980s, and then developed by Eppinger et al., 2013, which greatly improved its application field and use value. Now DSM has become a powerful tool for scholars to carry out management science research and help industrial design. The design structure matrix describes the interdependence between different elements in the form of a matrix (Browning, 2016). Due to its good operability, convenient calculation and excellent visualization effects, DSM has become a powerful tool for describing influence relations. In the beginning, DSM only used markers to indicate which elements have an influence relationship. With the development of research, the initial DSM gradually became a twodimensional matrix with numbers $0-1$ indicating the presence or absence of the relationship, and then developed to a numerical value indicating the size of the influence relationship. In addition, the original DSM is widely used in the fields of design and product research and development. After continuous promotion and development by scholars, the current DSM has become a common tool for research project progress, core team mining, iterative rework, and complexity reduction.

Existing clustering algorithms include: top-down or bottom-up algorithms, simulated annealing, genetic algorithms and other methods. In this paper, the top-down algorithm proposed by Thebeau is used. An element (component) will calculate the ADS value, and its function is similar to the bid value bid. During the calculation process, any element of the DSM has the same probability of being selected. Once an element is selected, the algorithm will calculate the bid value of that element and each class in the existing DSM, and then take the largest bid value.

\section{Model Building}

\subsection{Construct the relationship matrix between risk events and risk factors in QFD}

As shown in Figure 1(a), to establish a "risk event-risk factor" Quality Function Development (QFD). First, the product design expert determines the weight of each event demand and determines all the key risk factors related to these events. 


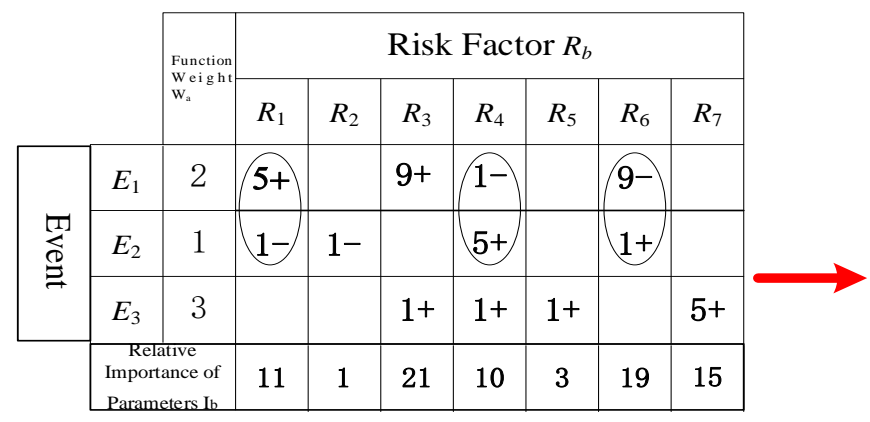

(a) Risk Factor QFD

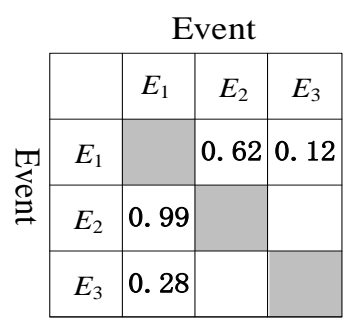

(b) Event DSM

Figure 1 Derivation of event DSM from Risk Factor QFD

Further, to determine the dependency matrix between QFD and risk factors, this article analyzes from two aspects (Tseng and Torng, 2011):

On the one hand, determine the degree of influence of changes in various risk factors on changes in related risk events. This article uses three values $(1,5,9)$ from expects to quantify the dependence between risk events and related risk factors, QFD $(a, b)$. The larger the value, the stronger the dependency between risk event $a$ and risk factor $R_{b}$, that is, the change of risk factor $R_{b}$ will cause the severity of risk event $a$ to change, and vice versa, the impact of $R_{b}$ change on risk event a weaker. Among them, each row of the QFD relationship matrix indicates which risk factors will be affected by the risk event $a$; each column of the relationship matrix indicates which risk events will be affected by the change of the risk factor $R_{b}$.

On the other hand, determine whether the change of risk factors has a positive correlation or a negative correlation on the impact of each risk event. A positive correlation means that if the value of a certain risk factor is larger, the value of risk events related to it will be higher; negative correlation. It means that if the value of a certain risk factor is larger, the value of risk events related to it is lower.

After determining the weight and relationship matrix of each risk event, the relative importance value of each risk factor can be obtained from formula (1):

$$
I_{b}=\sum_{a=1}^{n}\left(W_{a} \times Q F D(a, b)\right) \quad a=1,2, \ldots, n, \quad b=1,2, \ldots, M
$$

In the formula, $\mathrm{W}_{\mathrm{a}}$ represents the weight of each risk event, QFD $(\mathrm{a}, \mathrm{b})$ is the value of the row a and column $b$ in QFD, it represents the dependence between the risk event a and the risk factor $R_{b}$, and $n$ is the main product of the product. The number of risk events, $\mathrm{M}$ is the number of key risk factors. 


\subsection{Use QFD method to derive risk event DSM matrix}

As shown in Figure 1, QFD can deduce the strength of dependence between risk events and construct a corresponding DSM matrix of risk events. Regarding the dependence relationship between risk events, this article only considers the "restriction dependence relationship" between risk events, which means that if a certain risk factor $\mathrm{Rb}$ is positively correlated with a certain risk event, and at the same time it has a positive correlation with another risk event. Negative correlation, for $R_{b}$, there is a "restraint dependence relationship" between these two risk events. For example, in Figure 1(a), the change of risk factor $R_{1}$ increases the value of risk event $E_{1}$ (that is, positive correlation) and at the same time reduces the value of risk event $E_{2}$ (that is, negative correlation), then the risk event $E_{1}$ and $E_{2}$ are Constraint dependency; and the change of risk factor $R_{3}$ increases the values of risk events $E_{1}$ and $E_{3}$ (that is, both are positively correlated), there is no constraint dependency between these two risk events. For example, in the design of a thermos cup, if the thickness of the cup wall is increased, the insulation performance of the thermos cup can be improved, but the weight of the thermos cup will increase. Therefore, for the risk factor of the thickness of the cup wall, the insulation performance and the insulation cup. The weight of these two risk events has a constraint dependency relationship.

In R\&D project management, control dependencies are the key considerations of project managers. Project managers need to weigh the impact of changes in key risk factors on different risk events. Therefore, this article does not consider the impact of non-restricted dependencies on the project, and only considers these. To restrict the dependency relationship, the dependency strength W_DSM $(\mathrm{a}, \mathrm{m})$ between risk events a and $\mathrm{m}$ in the risk event DSM can be calculated by formula (2):

$$
W_{-} \operatorname{DSM}(a, m)=\sum_{b=1}^{M}\left(\omega_{b} \times I_{m \rightarrow a} \times Q F D(a, b)\right) / \sum_{b=1}^{M}\left(I_{b} \times Q F D(a, b)\right)
$$

$K$ is the number of key risk factors, $I_{m \rightarrow a}$ indicates whether the risk factor $\mathrm{R}$ has a restriction on the risk event a and $\mathrm{m}$. If there is no restriction, $I_{m \rightarrow a}=0$. On the contrary , $I_{m \rightarrow a}=1$.

In formula (2), the denominator represents the total dependency between risk event a and all related risk factors, and the numerator represents the sum of the dependency degrees in the risk event a and risk event $\mathrm{m}$ that have a restrictive relationship (that is, the restrictive dependency). Therefore, the dependence strength W_DSM (a, m) obtained from equation (2) is the percentage of the control dependence of risk event a and $\mathrm{m}$ in the total dependence of risk event a and all related risk factors, which represents the change of risk event $\mathrm{m}$ to the degree of influence of risk event $a$.

For example, based on the data in Figure 1(a), the dependency in the risk event DSM can be calculated from equation (2) (see Figure 1(b)):

W_DSM $(1,2)=(5 \times 11+1 \times 10+9 \times 19) /(1 \times 11+9 \times 21+1 \times 10+9 \times 19)=236 / 381=0.62$ 


$$
\begin{aligned}
& \text { W_DSM }(2,1)=(1 \times 11+5 \times 10+1 \times 19) /(1 \times 11+1 \times 1+5 \times 10+1 \times 19)=80 / 81=0.99 \\
& \text { W_DSM }(1,3)=(9 \times 21+1 \times 10) /(5 \times 11+1 \times 21+1 \times 10+9 \times 19)=31 / 257=0.12 \\
& \text { W_DSM }(3,1)=(1 \times 21+1 \times 10) /(1 \times 21+1 \times 10+1 \times 3+5 \times 15)=31 / 109=0.28
\end{aligned}
$$

This article assumes that a change in a certain risk event will cause all parameters related to the risk event to change, and only considers the mutual influence of risk events caused by the change of restricted parameters.

From equation (2), the risk event DSM can be obtained (see Figure 1(b)), W_DSM $(2,1)=0.6$ means that the change of related risk factors caused by the change of risk event $E_{1}$ has an impact intensity on risk event $E_{2}$ of 0.6 , In other words, the degree of risk event $E_{2}$ affected by the change of risk event $E_{1}$ is 0.6 . Therefore, from the direction of the risk event DSM column, it means that the parameter change caused by the change of the corresponding risk event $\mathrm{m}$ in this column affects other risk events; from the row direction, it means that the corresponding risk event a of this row is affected by other risk events. The degree of impact caused by the change of the risk event. In other words, each column of the risk event DSM represents the impact of the output, that is, the degree of influence of the column risk event $m$ on the row risk event $a$; each row represents the influence of the input, that is, the degree of influence of the row risk event a by the column risk event $m$. The upper triangle of W_DSM $(\mathrm{a}, \mathrm{m})$ represents the impact of the change of risk event corresponding to the column on risk event, and the lower triangle represents the impact of the change of risk event corresponding to the row by the change of risk event.

\subsection{Construction and Analysis of the Network Model of Risk Dependence Between Projects}

There are complex interactions between risk events and projects. On the one hand, there are correlations between risk events, and on the other hand, there are dependencies between projects and risk events. To this end, this article uses the "risk event-project" multi-domain matrix (MDM) to push to the project DSM. MDM is an extension of the DSM model, which can simultaneously represent two DSM models in different fields. MDM reflects the dependencies between cross-domain elements, and it provides a useful analysis tool for identifying the relationships between elements in different domains.

As shown in the figure, the diagonal position in the "risk event-project" MDM represents the risk event DSM (upper left corner) and the project DSM (lower right corner), and the off-diagonal position represents DMM (lower left corner). Among them, the risk event DSM represents the interdependence between risk events. The "columns" of the matrix indicate which risk events will affect which risk events occur. "Off-diagonal" indicates which risk events will be affected by the risk event, and the non-diagonal value indicates the probability of one risk event causing another risk event; project DSM indicates the dependency relationship between projects; "risk event-project" DMM indicates the occurrence of risk events and the dependency relationship between projects, this paper uses a number between 1-3, the larger the value, the stronger the dependency relationship, and its value is determined by expert judgment. 


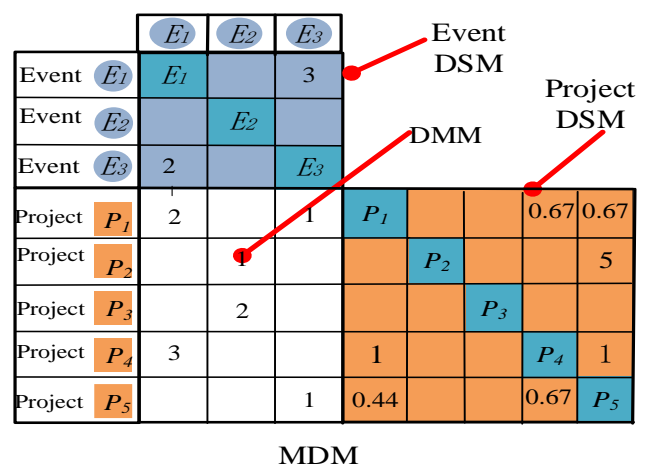

Figure 2 the Network Model of Risk Dependence Between Projects

A basic model of MDM analysis of inter-domain dependencies between elements is established. If the dependency $E_{-} D S M\left(E_{a}, E_{m}\right)$ between the risk event $E_{a}$ and $E_{m}$ and the dependency $\operatorname{DMM}\left(P_{M}, E_{m}\right)$ between the risk event $E_{m}$ and the project $P_{M}$ is known, the strength of the dependency relationship between $P_{M}$ and $P_{A}$ (that is, the probability of impact) in the project DSM can be obtained by formula (4):

$$
P_{-} \operatorname{DSM}\left(P_{A}, P_{M}\right)=\sum_{a=1}^{n}\left(\operatorname{DMM}\left(P_{A}, E_{a}\right) \times \sum_{n=1, n \neq a}^{n}\left(\operatorname{DMM}\left(P_{M}, E_{m}\right) \times E_{-} D S M\left(E_{a}, E_{m}\right)\right)\right)
$$

$a, m=1,2, \cdots, n, A, M=1,2, \cdots, N, \quad n$ and $N$ represent the number of risk events and projects in the project, respectively.

Project DSM is a static DSM model, and clustering method is usually used to optimize it (Eppinger and Browning, 2012). Clustering method can divide complex projects into several different categories or modules. Usually, each module is composed of projects with high risk correlation (Yang et al, 2014). Make the degree of dependence within the class closer, and reduce the degree of dependence between classes, achieving the goal of reducing management complexity. As shown in the figure, usually, the goal of DSM clustering is to reduce the dependence of the external class or increase the dependence of the internal class by changing the position of the element in the DSM, that is, through the clustering operation, it has a strong interaction. Most of the elements of DSM cluster into the same category, and the interaction strength (dependency) between the elements outside the category should be as weak as possible. According to different application fields, the existing DSM clustering objectives include: minimum description length (minimum description length), maximize module measurement or minimize coordination costs, etc. 


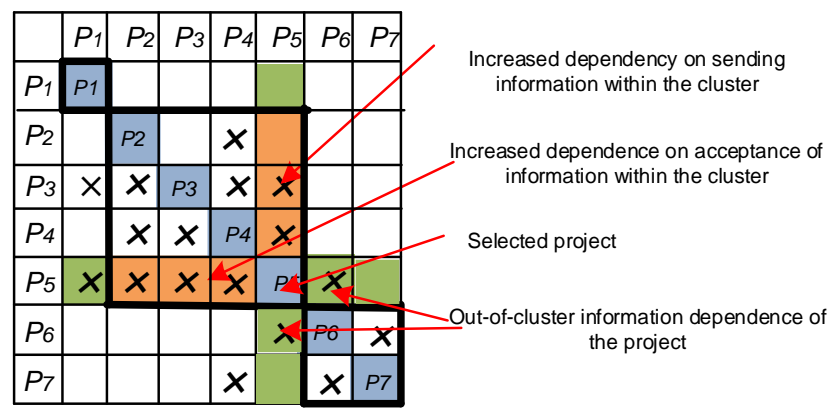

Figure 3 Intensity of dependence between projects

In order to maximize the interaction strength between the elements within the class, this paper proposes to maximize the ADS (added average dependency strength) as the clustering objective function, and use this index to measure whether the selected element should be added. For a certain category, ADS represents the ratio of the sum of the dependence of the corresponding row and column of the selected element in a certain category to the number of newly added cells of the category, as shown in the figure, if the selected element is selected (Project P1) and existing classes (P2, P3, P4) are grouped into one class, that is, element P5 is added to the existing classes (P2, P3, P4), and ADS is the sum of the in-class row and column dependencies corresponding to element P5. The ratio of (the dark part of the second category in the figure) to the number of newly added units of this category (the number of newly added units of this category), ADS can be calculated by formula (5):

$$
\max \operatorname{ADS}\left(\text { cluster }_{\alpha}\right)=\frac{\left(\sum_{g=t_{\alpha}}^{t_{\alpha}-1} C_{-} \operatorname{DSM}\left(g, t_{\alpha}\right)+\sum_{h=t_{\alpha}}^{t_{\alpha}-1} C_{-} \operatorname{DSM}\left(t_{\alpha}, h\right)\right)^{\gamma}}{2\left(c l_{\alpha}-1\right)}
$$

$c l_{\alpha}$ is the scale of $\alpha, r_{\alpha}$ and $t_{\alpha}$ are the numbers of the first and last elements in the class, respectively, $\gamma$ is the coefficient.

The interaction strength between the project and other projects is calculated by equation (5), that is, the dependence strength between the projects, which facilitates the grouping of the projects with strong correlation, so as to achieve better monitoring and management of the project set with high risk correlation.

\section{Conclusion and Future Work}

Current projects are faced with various risks. How to group projects with high risk correlations together to facilitate management has become a hot topic today. Therefore, this article first constructs the dependency matrix between risk events and risk factors, and uses the QFD method to derive the risk event DSM matrix, and obtains the dependency relationship model between risk events; then, constructs the risk dependency relationship between the projects, through risk. The event-project MDM derives the project DSM; finally, the project DSM is optimized and analyzed by the clustering method, and the 
dependence intensity between projects is calculated, which is convenient for monitoring and managing the project set with high risk correlation.

In the future, it is mainly through big data methods to collect risk events and risk factors to facilitate cluster management of projects with a high degree of risk correlation. In addition, you can continue to optimize the clustering algorithm to facilitate monitoring and management when there are many items.

\section{Acknowledgments}

This study was supported by the National Natural Science Foundation of China (No.71872011 and No.71929101).

\section{References}

Aerts, G., Dooms, M., Haezendonck, E., 2016. Knowledge transfers and project-based learning in large scale infrastructure development projects: An exploratory and comparative ex-post analysis. International Journal of Project Management 35(3), 224-240.

Browning, T. R., 2016. Design structure matrix extensions and innovations: a survey and new opportunities. IEEE Transactions on Engineering Management, 63(1), 1-26.

Eppinger, S. D, Joglekar, N. R, Olechowski, A., 2013. Improving the systems engineering process with multi-domain mapping,15th International DSM Conference, Australia, Melbourne, August 29-30, 63-70.

Eppinger, S. D. , \& Browning, T. R., 2012. Design structure matrix methods and applications. Mit Press Books, 1.

Ghapanchi, A. H., Tavana, M., Khakbaz, M. H. et al., 2012. A methodology for selecting portfolios of projects withinteractions and under uncertainty. International Journal of Project Management 30 (7): 791-803.

Kim, W., Kim, Y. M., Kim, T. H. et al., 2018. Multi-dimensional portfolio risk and its diversification: A note. Global Finance Journal 35: 147-156.

Neumeier, A., Radszuwill, S., Garizy, T. Z., 2018. Modeling project criticality in IT project portfolios. International Journal of Project Management 36(6): 833-844.

Tseng, C. C., Torng, C. C., 2011. Prioritization determination of project tasks in QFD process using design structure matrix. Journal of Quality 18 (2): 137-146.

Yang, Q., Yao, T., Lu, T., Zhang, B., 2014. An overlapping-based design structure matrix for measuring interaction strength and clustering analysis in product development project. IEEE Transactions on Engineering Management, 61(1):159-170.

Contact: Qing Yang, University of Science \& Technology Beijing, School of Economics and Management, No.30 Xueyuan Road, Haidian District, Beijing 100083, China, 86+13911463353, yangqing@manage.ustb.edu.cn. http://dsem.ustb.edu.cn/jgen/a/Faculfy_List/20141009/124.html 